\title{
Eubacterium rectale contributes to colorectal cancer initiation via promoting colitis
}

\author{
Yijia Wang ${ }^{1+}$, Xuehua Wan ${ }^{2+}$, Xiaojing Wu${ }^{1}$, Chunze Zhang ${ }^{1}$, Jun Liu ${ }^{1 *}$ (D) and Shaobin $\mathrm{Hou}^{3 *}$
}

\begin{abstract}
Background: Inflammatory bowel disease caused by microbial dysbiosis is an important factor contributing to colorectal cancer (CRC) initiation. The 'driver-passenger' model in human gut microbial dysbiosis suggests that 'driver' bacteria may colonize with low relative abundance on tumor site but persistently induce chronic change in normal intestinal epithelium and initiate CRC. They are gradually replaced by 'passenger' bacteria later on, due to their low adaptability to the on-tumor site niche.

Results: To reveal site-specific bacterial taxon markers in CRC patients, we analyzed the gut mucosal microbiome of 75 paired samples of on-tumor and tumor-adjacent sites, 75 off-tumor sites, and 26 healthy controls. Linear discriminant analysis of relative abundance profiles revealed unique bacterial taxon distribution correlated with specific tumor sites, with Eubacterium having the distribution characteristic of potential driver bacteria. We further show that Eubacterium rectale endotoxin activates the transcription factor NF-KB , which regulates multiple aspects of innate and adaptive immune responses in normal colon epithelial cells. Unlike the 'passenger' bacterium Fusobacterium nucleatum, E. rectale promotes dextran sodium sulfate-induced colitis in Balb/c mice.
\end{abstract}

Conclusions: Our findings reveal that E. rectale functions as a 'driver' bacterium and contributes to cancer initiation via promoting inflammation.

Keywords: Eubacterium rectale, Colorectal cancer,'driver-passenger' model, Colitis, Microbiota

\section{Background}

The adenoma-carcinoma sequence indicates that the accumulation of genetic and epigenetic alterations induces malignant epithelial cell proliferation, leading to colorectal cancer (CRC) [1]. Recent studies suggest that gut microbial dysbiosis results in colitis which contributes to initiation and development of CRC [2]. It is hardly to obtain samples from a same patient who went through the process from inflammatory bowel disease

\footnotetext{
*Correspondence: junliu_sci@163.com; Shaobin@hawaii.edu

${ }^{\dagger}$ Yijia Wang and Xuehua Wan contribute equally to this work

1 Laboratory of Oncologic Molecular Medicine, Tianjin Union Medical Center, Nankai University, No. 190 Jieyuan Rd., Hongqiao district,

Tianjin 300121, China

${ }^{3}$ Advanced Studies in Genomics, Proteomics, and Bioinformatics,

University of Hawaii At Manoa, 2538 McCarthy Mall, Snyder Hall, Honolulu, HI 96822, USA

Full list of author information is available at the end of the article
}

(IBD) to CRC, so distribution of gut bacteria in on-tumor sites and off-tumor sites of CRC patients is usually used to investigate bacteria associated with initiation of CRC by promoting inflammation. Deep sequencing-based 16S rRNA profiling can detect biodiversity of gut microbiome and reveal the relationship between gut microbiome and sporadic CRC. Microbial metabolites, such as butyrate and hydrogen sulfide [3, 4], as well as bacterial pathogen-associated molecular patterns (PAMP), such as lipopolysaccharide (LPS) [2], are known for triggering proinflammatory cascades that lead to adenoma-carcinoma sequence.

A variety of gut bacteria is more abundant on CRC tissue surface than on normal large intestinal surface. For example, Fusobacterium nucleatum in tumor samples shows high abundance but is almost absent from normal intestinal surface [5]. However, F. nucleatum

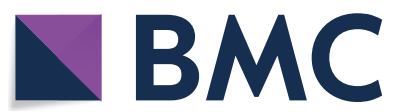

(c) The Author(s) 2021. This article is licensed under a Creative Commons Attribution 4.0 International License, which permits use, sharing, adaptation, distribution and reproduction in any medium or format, as long as you give appropriate credit to the original author(s) and the source, provide a link to the Creative Commons licence, and indicate if changes were made. The images or other third party material in this article are included in the article's Creative Commons licence, unless indicated otherwise in a credit line to the material. If material is not included in the article's Creative Commons licence and your intended use is not permitted by statutory regulation or exceeds the permitted use, you will need to obtain permission directly from the copyright holder. To view a copy of this licence, visit http://creativecommons.org/licenses/by/4.0/. The Creative Commons Public Domain Dedication waiver (http://creativecommons.org/publicdomain/zero/1.0/) applies to the data made available in this article, unless otherwise stated in a credit line to the data. 
may not promote CRC [6], because its high abundance in tumor samples may be caused by alterations of gut microenvironment [7]. F. nucleatum does not exhibit pro-tumorigenic or proinflammatory activities in preclinical models of colon carcinogenesis [6] and has high abundance in CRC samples, but not colorectal carcinoma [8]. Some bacteria, like F. nucleatum, cannot breach the intact healthy colon wall and colonize. However, they have a competitive advantage in tumor environment with rupture and bleeding of the colonic epithelium [7]. Thus, these bacteria are 'passenger' bacteria based on the 'driver-passenger' model proposed by Tjalsma et al. [9].

'Driver' bacteria initiate CRC by inducing IBD in epithelial cells. Meanwhile, they are gradually substituted by 'passenger' bacteria because gut microenvironment changes during tumor development. Many 'driver' bacteria are prone to be ignored if their abundances in CRC tissue are used as the only criterion. For example, Bacteroides fragilis is a potential 'driver' bacterium. It is a common colonic commensal which colonizes the majority of human guts. A subset of them is identified as enterotoxigenic $B$. fragilis (ETBF) which secrets a $20 \mathrm{kDa}$ metalloprotease toxin (BFT). BFT cleaves E-cadherin, stimulates cell proliferation, and promotes IBD [10, 11]. Furthermore, CRC microbiomes analysis showed that Bacteroides are less abundant in CRC tissue than in adjacent non-malignant tissue [5]. Thus, as a potential 'driver' bacterium, $B$. fragilis secrets a toxin that may contribute to IBD that leads to CRC initiation, but it has relatively low growth competition on CRC epithelium. Bacterial taxon markers associated with off-tumor sites may serve as potential driver bacteria for CRC initiation [9]. Further identification and characterization of driver bacteria and their pathogenic activities will pave the way to CRC prevention therapies.

To identify CRC-associated gut microbiome profiles and potential driver bacteria that initiate CRC, we performed $16 \mathrm{~S}$ ribosomal RNA gene sequencing on gut mucosal microbiome of paired samples of tumor and tumor-adjacent mucosae, off-tumor sites and healthy controls. The gut microbiome of tumor mucosae, but not that of tumor-adjacent mucosae and healthy control, shows lower alpha-diversity and distinct bacterial taxa compared with that at off-tumor sites. Linear discriminant analysis Effect Size (LEfSe) revealed specific bacterial taxa associated with tumor and tumor-adjacent mucosae, off-tumor sites and healthy controls, respectively. Moreover, the relative abundance of Eubacteriaceae is higher on off-tumor sites than tumor mucosae. Using Eubacterium rectale as an example, we showed the effect of $E$. rectale LPS on colon epithelium cells in vitro and that $E$. rectale promotes IBD in vivo.

\section{Materials and methods \\ Reagents}

All cell culture media, trypsin and antibiotics were purchased from Gibco (Grand Island, NY, USA), and FBS was purchased from HyClone (Logan, UT, USA). DAPI, lysosyme, proteinase $\mathrm{K}$, DNase and RNase were purchased from Sigma-Aldrich (St Louis, MO, USA). Immobilon membranes were purchased from Merck Millipore (Bedford, MA, USA). ECL Plus substrate was purchased from CWBio (Beijing, China). Nuclear and cytoplasmic protein extraction kit was purchased from Beyotime (Shanghai, China). ZR Fungal/Bacterial DNA Kit was purchased from Zymo Research Corp. (Irvine, CA, USA). Qualitative fecal occult blood detection kit was purchased from Beijing Leagene Biotechnology Co., Ltd. (Beijing, China). Limulus Amebocyte Lysate (catalogs: T7572) was purchased from Solarbio (Beijing, China).

Rabbit anti-nuclear factor kappa B (anti-NF-кB) p65 antibody (catalogs: SAB4502610), rabbit anti-Histone H3 antibody (catalogs: SAB4500354), rabbit anti-IKK $\alpha$ antibody (catalogs: SAB4500257), rabbit anti-ІкB $\alpha$ antibody (catalogs: SAB1305978), rabbit anti- $\beta$-actin antibody (catalogs: SAB2100037), goat anti-rabbit IgG-peroxidase (catalogs: A0545) and goat anti-rabbit IgG FITC (catalogs: AP132F) were purchased from Sigma-Aldrich (St Louis, MO, USA).

\section{Sample collection and preparation}

Intestinal microflora samples were collected from 75 patients and 26 health people in Tianjin Union Medical Center. Cotton swab was used to dip on intestinal surface of colorectal cancer tissues collected from patients. Three distinct locations from the same tissue of a given cancer patient were collected, including tumor position $(\mathrm{T})$, para-tumor epithelia position $(\mathrm{P})$ and normal epithelia position $(\mathrm{N})$. Intestinal microflora samples of healthy people were collected when people were diagnosed for colonoscopy and determined as healthy people $(\mathrm{H})$. These samples were rinsed in $1 \mathrm{ml}$ physiological saline. Then, $200 \mu \mathrm{l}$ of these solutions were used for Bacterial DNA extraction. Bacterial DNA of these samples was extracted using ZR Fungal/Bacterial DNA Kit according to the manufacturer's instructions.

\section{S RNA sequencing and bioinformatic analysis}

The 16S ribosomal RNA amplicon libraries were constructed according to the Illumina manufactory manual. Briefly, the following primers were used to amplify the V3-V4 region of $16 \mathrm{~S}$ rRNA gene: forward primer, $5^{\prime}$ TCG TCGGCAGCGTCAGATGTGTATAAGAGACAGCCT ACGGGNGGCWGCAG and reverse primer $5^{\prime}$ GTC TCGTGGGCTCGGAGATGTGTATAAGAGACAGGA CTACHVGGGTATCTAATCC. 
The amplified DNA library was subsequently purified using AMPure XP beads (Beckman Coulter, USA) and quantified using Quant-iT PicoGreen dsDNA assay kit (Thermo Fisher, USA). The paired-end sequencing reads $(2 \times 300 \mathrm{bp})$ were generated on Illumina MiSeq platform, according to the Illumina standard protocol. Quality control and filtering of raw sequences were carried out using FastQC (https://www.bioinformatics.babraham. ac.uk/projects/fastqc/). The filtered paired-end reads were assembled using PandaSeq [33]. The assembled sequences were loaded on QIIME pipeline (qiime.org) [34] for de novo OTU picking, taxonomic assignment, and diversity analyses. Usearch was used within QIIME to detect and remove de novo chimera. Rarefaction was performed using alpha_rarefaction.py in QIIME pipeline. De novo OTUs were picked based on sequence similarity (97\%) within the assembled sequences. Next, taxonomy was assigned to OTU representative sequences that were picked for each OTU. To identify differential abundance of bacterial phylo associated with specific sites of CRC patients and healthy people, we applied LEfSe [35].

\section{Bacterial strains and culture conditions}

E. rectale ATCC 33656 and F. nucleatum subsp. Nucleatum ATCC 25586 were both purchased from Biobw (Beijing, China). E. rectale was grown anaerobically in PYG medium (ATCC medium 1527) composing of 0.5\% peptone, $1 \%$ yeast extract, $0.5 \%$ tryptone, $0.01 \%$ resazurin (Sigma), $0.0008 \% \mathrm{MgSO}_{4}, 0.0008 \%$ anhydrous $\mathrm{CaCl}_{2}$, $0.004 \% \mathrm{~K}_{2} \mathrm{HPO}_{4}, 0.004 \% \mathrm{KH}_{2} \mathrm{PO}_{4}, 0.002 \% \mathrm{NaCl}, 0.04 \%$ $\mathrm{NaHCO}_{3}, 0.0001 \%$ (v/v) Vitamin K1 (Sigma), 0.05\% L-cysteine-HCL (Sigma). The pH was adjusted to 7.0. F. nucleatum ATCC 25586 was maintained anaerobically in Columbia broth (BD Biosciences). PYG medium and Columbia broth were both boiled to remove oxygen and placed in anaerobic bottles. Argon gas was added to the bottles. Then the bottles were sealed by a cap with a rubber septum before being autoclaved for $20 \mathrm{~min}$ at $121^{\circ} \mathrm{C}$.

\section{Mouse models of bacterial colonization}

Colitis was experimentally induced by administration of $2.5 \%$ Dextran sodium sulfate (DSS) in the drinking water of 7-week-old Balb/c mice for 7 days. Afterwards, the mice were allowed to drink water without DSS until the end of experiment. After 2 days of DSS drinking, mice were inoculated with $E$. rectale or $F$. nucleatum by coloclysis with $1 \times 10^{7}$ colony forming units (CFU) in $200 \mu \mathrm{l}$ PBS. Briefly, intraperitoneal injection of $4 \%$ chloral hydrate $(10 \mu \mathrm{l} / \mathrm{g})$ was used to deeply anesthetize mice. The mice were kept to prone position. Colon was inserted with paraffin oil applied plastic hose ( $2 \mathrm{~mm}$ internal diameter) through anus. When the top of hose was inserted $4 \mathrm{~cm}$ from anus, the mice were kept to handstand. The hose was pulled out after bacterium was injected. Anus was pressed with cotton swab within $1 \mathrm{~min}$, and the mice were put back to cage. Mice were separated into 6 groups: (Water) control group, water drinking and no bacterial inoculation; (DSS) DSS drinking and no bacterial inoculation; (E. rectale + water) water drinking and $E$. rectale inoculation; (F. nucleatum + water) water drinking and $F$. nucleatum inoculation; (E. rectale + DSS) DSS drinking and E. rectale inoculation; (F. nucleatum +DSS) DSS drinking and F. nucleatum inoculation. Each group consisted of five mice. The mice were monitored and weighed daily until they had lost $>20 \%$ of their initial body weight, And upon being sacrificed, the colon and spleen were both harvested for determination of colon length, histological examination and spleen weight. Stool was collected and measured daily for the presence of occult and gross blood. Occult blood was measured by a qualitative fecal occult blood detection kit.

\section{Histological examination}

Harvested colons were cleaned in physiological saline solution to remove fecal residue. After $24 \mathrm{~h}$ of fixation in $10 \%$ buffered formalin, the colons were embedded in paraffin and sectioned. Then the tissues were stained with hematoxylin and eosin (H\&E). Images of histology slides were taken by an Olympus IX51 microscope. Histology of colon was scored on a scale of $0-5$ where $0=$ normal and $5=$ severe inflammation and complete loss of surface and crypt epithelium.

\section{Isolation of bacterial endotoxins}

Endotoxic LPSs were isolated from bacteria using the hot phenol-water method as previously described [36]. In brief, bacterial pellets were digested with lysozyme. Then, cell lysate was incubated with DNase and RNase to digest nucleic acids. 90\% phenol extraction procedure was used. The water layer was dialyzed against deionized water and digested with proteinase $\mathrm{K}$. Then the solution was dialyzed again. The purity of the phenol extract was tested by the detection of nucleic acid $(260 \mathrm{~nm})$, coomassie brilliant blue stained protein $(595 \mathrm{~nm})$. Isolated LPSs were also detected by SDS-PAGE. Finally, LPSs were lyophilized using Freeze Dry System (FD-1A-80, BILON Ltd., Shanghai, China). The biological effectiveness of isolated LPSs was tested in Limulus Amebocyte Lysate assay according to the manufacturer's instructions.

\section{LPS treatment to human normal colon epithelial cells}

The human normal colon epithelial cell lines NCM460 and $\mathrm{HCoEpiC}$ were both purchased from Tong Pai Technology, Inc. (Shanghai, China). These cells were cultured in RPMI 1640 medium supplemented with 10\% FBS, 
$100 \mu \mathrm{g} / \mathrm{mL}$ streptomycin, and $100 \mathrm{U} / \mathrm{mL}$ penicillin. LPS was dissolved in RPMI 1640 medium and vortexed for 10 min by ultrasonic bath (Shenglan Ltd., Jiangsu, China) before use. A concentration series of LPS dilutions was used to treat cells for $2.5 \mathrm{~h}$.

\section{Western blotting}

For detection of NFkB p65 expression in nuclei, nuclear proteins were isolated from harvested cells using a nuclear and cytoplasmic protein extraction kit and following the manufacturer's protocol. Western blotting was carried out as previously described [37]. Briefly, protein samples were suspended in SDS loading buffer and boiling for $5 \mathrm{~min}$. Then $10 \mu \mathrm{g}$ protein was separated by SDS-PAGE and transferred to immobilon membranes by semi-dry blotting method. The membranes were probed with antibodies using standard techniques. Finally, the protein bands were visualized using ECL Plus and exposed film. Each assay was carried out in triplicate.

\section{Immunocytochemistry analysis}

Immunofluorescence staining was used to analyze the location of NFkB p65 expression. The procedure was carried out as previously described [37]. In brief, cells were washed with PBS for three times, fixed in $10 \%$ formalin at room temperature for $20 \mathrm{~min}$, treated with $0.5 \%$ Triton $\mathrm{X}-100$ for $5 \mathrm{~min}$ at $4{ }^{\circ} \mathrm{C}$ and blocked with $5 \%$ normal goat serum overnight at $4{ }^{\circ} \mathrm{C}$. Then the slides were incubated with primary antibody for $1 \mathrm{~h}$ at $37^{\circ} \mathrm{C}$. After washing with PBS, the slides were incubated with FITCconjugated secondary antibody and DAPI simultaneously for $1 \mathrm{~h}$, at $37{ }^{\circ} \mathrm{C}$. Subsequently, the slides were washed with PBS and then sealed. The slides were photographed immediately using a fluorescence microscope (Olympus, Tokyo, Japan).

\section{Statistical analysis}

All data represent mean \pm standard deviation. Statistical analysis was carried out by Student's $t$ test using SPSS software. P value $<0.05$ was considered statistically significant.

\section{Results}

Decreased alpha-diversity and altered overall microbial composition in location of severe lesion intestinal epithelium

To reveal microbiome profile differences among gut mucosal phenotypes, we performed $16 \mathrm{~S}$ ribosomal RNA gene sequencing on biopsy samples collected from ontumor sites $(n=75)$, tumor-adjacent sites $(n=75)$, and off-tumor sites $(\mathrm{n}=75)$ of CRC patients, and healthy people $(n=26)$ at Tianjin Union Medical Center (Table 1). In the discovery cohort, severity of pathological changes in intestinal epithelium was strongly associated with a decrease of intraindividual diversity of on-tumor sties compared to off-tumor sites, as measured by Shannon diversity index $(\mathrm{p}<0.05)$ (Fig. 1a). Alpha-diversity in microbiome profiles may be affected by individual difference. It has been reported that Shannon diversity index is similar in fecal samples of normal, adenoma and cancer group [12], but it is also found that bacterial diversity of gut mucosal samples is lower in CRC group than normal group [13]. To assess the overall diversity of microbiome profiles, we performed principal component analysis (PCA) based on genera abundances in different mucosal sites from CRC patients and healthy people. Figure $1 \mathrm{~b}$ showed the microbial composition differences in different mucosal sites of patients and healthy controls.

\section{Bacterial differential abundances in different mucosal sites of patients and healthy controls}

Using the reference Greengenes database, clustered operational taxonomic units (OTU) were assigned for bacterial phylotypes. Consistent with previous findings [13], we observed the same major bacterial phyla across the different mucosal sites of patients and healthy controls, including Firmicutes, Bacteroides, and Proteobacteria. To identify differentially abundant taxa in mucosal phenotypes as site-specific bacterial taxon markers, we performed linear discriminant analysis (LDA) on the microbial compositions of the different mucosal sites from CRC patients and healthy people (LDA score $>2.5$, $\mathrm{p}$ value $<0.05)$. No significant changes were observed at

Table 1 Summary information of CRC patients and healthy people

\begin{tabular}{|c|c|c|c|c|}
\hline \multirow[t]{2}{*}{ Position } & \multicolumn{2}{|l|}{ CRC patients (75) } & \multicolumn{2}{|c|}{ Healthy volunteers (26) } \\
\hline & Rectum & Colon & Rectum & Colon \\
\hline No & 43 & 32 & 20 & 6 \\
\hline Male/female & $29 / 14$ & $19 / 13$ & $14 / 6$ & $3 / 3$ \\
\hline Age (mean, range) & $63.9(29-81)$ & $62.8(35-82)$ & $50.2(21-71)$ & $56.5(48-68)$ \\
\hline Dukes & $A(3), B(23), C(17)$ & $A(4), B(14), C(14)$ & & \\
\hline
\end{tabular}

A total of 101 subjects were included in this study: 75 individuals in the CRC group and 14 individuals in healthy group. The mean age of the subjects was $63.4 \pm 11$ years in CRC and $51.7 \pm 11.9$ years in healthy group 


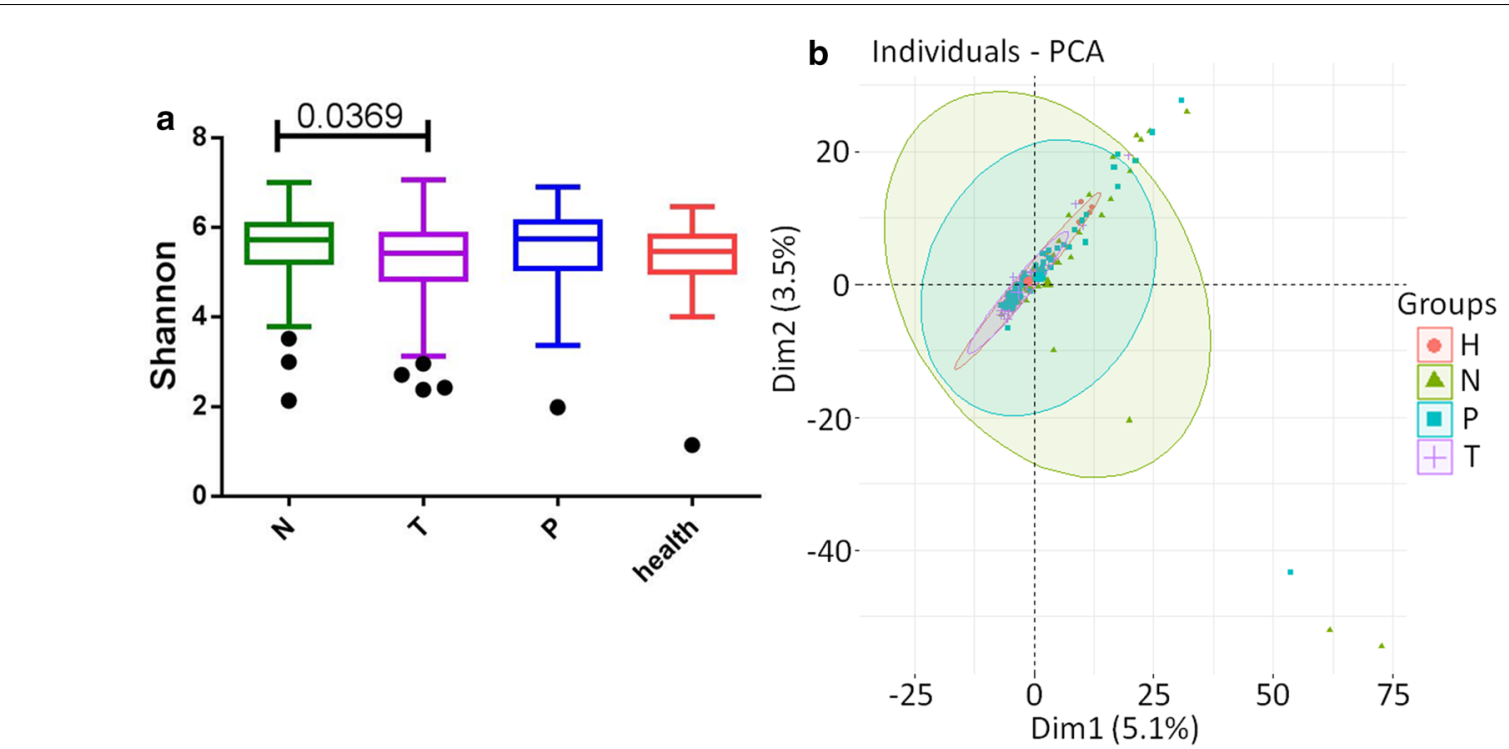

Fig. 1 Gut microbiome biodiversity of CRC patients and healthy controls. a Shannon diversity index was significantly reduced in on-tumor sites compared to off-tumor sites. P value was tested using one-tailed student's t test. $\mathbf{b}$ PCA analysis showed that the overall gut microbiota composition was different among on-tumor sites, adjacent-tumor sites, off-tumor sites, and healthy controls

phylum level among different mucosal sites from CRC patients and healthy people. In total, $15,5,8$, and 15 families were identified to be associated with on-tumor site, adjacent-tumor site, off-tumor site, and healthy control, respectively (Fig. 2). At family level, Fusobacteriaceae showed the strongest correlation with on-tumor site $(\mathrm{LDA}$ score $=4.86$, $\mathrm{p}$ value $=3.28 \mathrm{E}-07)$ (Fig. $2 \mathrm{a})$, consistent with previous findings [5]. Bradyrhizobium (LDA score $=3.71$, $\mathrm{p}$ value $=1.32 \mathrm{E}-11$ ) and Corynebacteriaceae $(\mathrm{LDA}$ score $=3.49$, $\mathrm{p}$ value $=1 \mathrm{E}-04)$ showed the strongest correlation with tumor-adjacent sites of CRC patients, distinct different from the taxon markers for on-tumor sites (Fig. 2b). Erysipelotrichaceae (LDA score $=4.02, \mathrm{p}$ value $=0.03)$ and Ruminococcaceae $($ LDA score $=4.84, p$ value $=0.03)$ showed the strongest correlation with offtumor sites and healthy controls, respectively (Fig. $2 \mathrm{c}$ and d). Our data suggest that microbial compositions in different mucosal sites and healthy controls show distinct microbial profiles. In addition, bacteria associated with off-tumor site, including the family Eubacteriaceae, may serve as driver bacteria that initiate CRC.
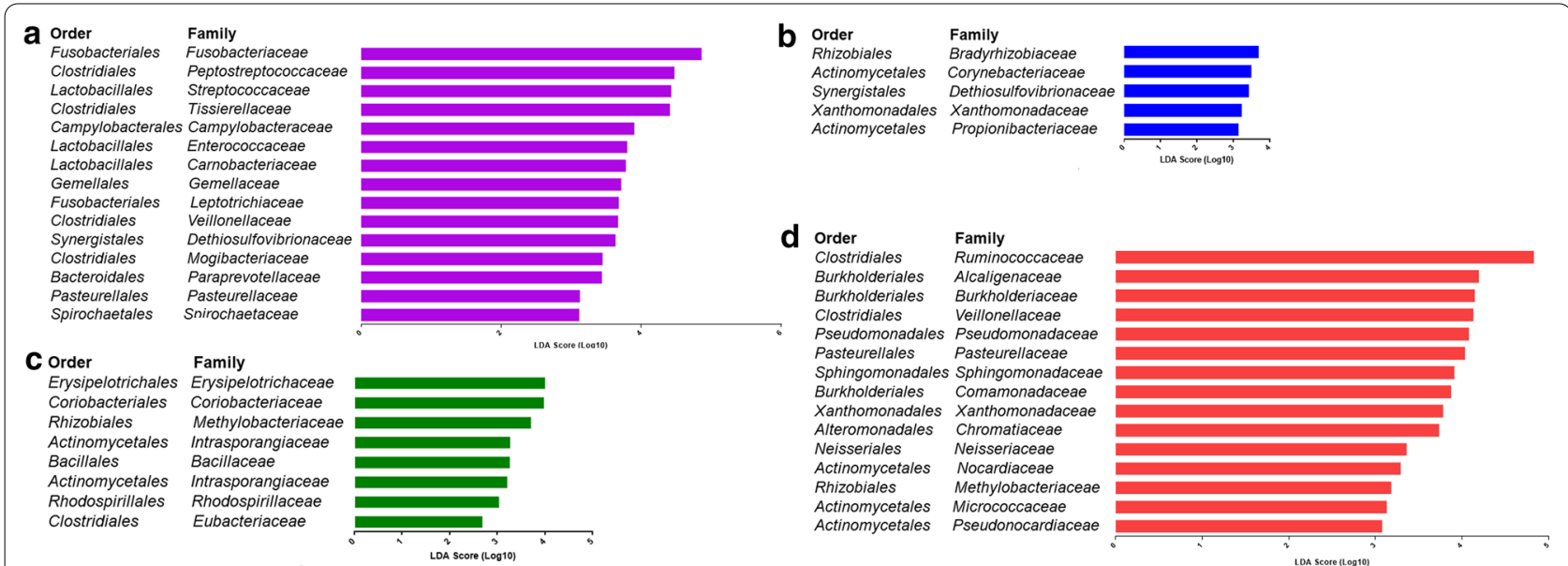

Fig. 2 LDA analysis showed the site-specific bacterial markers in on-tumor sites (a), adjacent-tumor sites (b), and off-tumor sites (c) of CRC patients and healthy controls (d) 


\section{E. rectale is a potential driver bacterium for CRC initiation} Among the eight bacterial families associated with offtumor site of CRC patients, the family Eubacteriaceae contains a butyrate-producing genus Eubacterium. Here we chose E. rectale as an example to examine the role of Eubacterium in CRC initiation. As shown in Fig. 3a, abundances of $E$. rectale and $F$. nucleatum were calculated by QIIME. As a typical bacterium associated with $\mathrm{CRC}$, according to previous reports [8], F. nucleatum exhibits high abundance in intestinal tract of CRC patients and is practically nonexistent in samples from healthy people. Furthermore, tumor location has the highest abundance of $F$. nucleatum among all three locations of patients' intestine, suggesting that $F$. nucleatum is suitable to grow in the tumor microenvironment. It has been reported that $E$. rectale is commonly distributed in healthy intestinal tract, and is less abundant in CRC patients [14]. Our results showed that there was lower abundance of $E$. rectale in on-tumor site of CRC patients than off-tumor site, as well as that E. rectale exhibits higher abundance in on-tumor site of CRC patients than healthy people samples. Local intestinal microenvironment of off-tumor sites of CRC patients is expected to be more like that of healthy people samples than that of ontumor sites of CRC patients. Higher abundance of E. rectale in off-tumor site compared with healthy people may be due to other reasons, such as eating habits or individual difference. This difference in E. rectale abundance

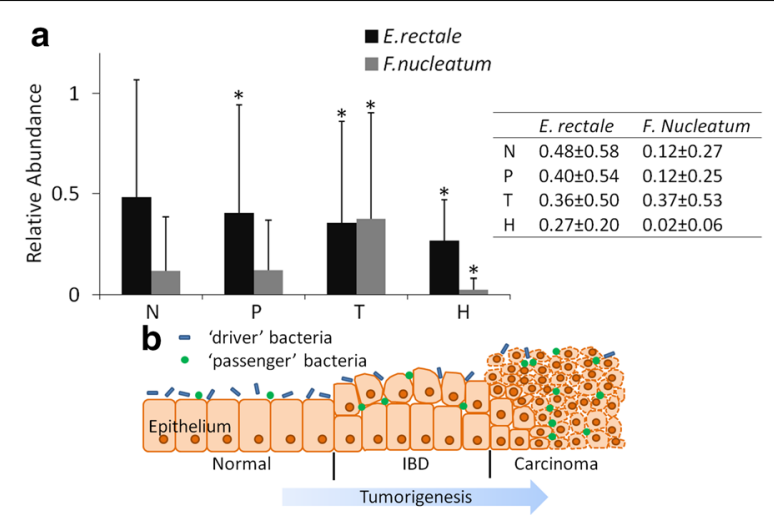

Fig. 3 Abundance of bacteria in patient samples and diagrammatic sketch of colon epithelium. a Abundance of E. rectale and $F$. nucleatum at different sites on the colon in samples of 75 patients and 26 health people is shown as mean \pm STD. "N" represents normal epithelia position of patients, " $P$ " represents para-tumor epithelia position of patients, "T" represents tumor surface of patients, " $\mathrm{H}$ " represents healthy people. Other groups were compared with group "N" separately using Student's t test. ${ }^{*} P<0.05$ represents a significant difference from the value in the " $\mathrm{N}$ " group. $\mathbf{b}$ During tumorigenesis, abundance of 'driver' bacteria, which contributed to tumor initiation, decreased gradually owing to the high competition from the 'passenger' bacteria in IBD or tumor niche may also be due to its presence in these people's intestinal tract before CRC onset, which is also one reason for CRC initiation. Therefore, we speculate that $E$. rectale may be a 'driver' bacterium, and next investigated the role $E$. rectale in CRC initiation (Fig. 3b).

\section{E. rectale promotes colitis in DSS mouse model of colitis-associated cancer}

DSS mouse model of colitis was used to examine whether E. rectale promotes colitis which contributes to initiation of CRC. Treatment of mice in each group was shown in Fig. 4a. Histopathological analysis of H\&E stained colon sections (Fig. 4b and c) revealed that, compared to the normal colonic tissue of water drinking group, DSS exposure led to segmental regions of intestinal mucosa rupture, marked acute inflammatory cell infiltration, crypt abscesses, and early architectural distortion. E. rectale but not $F$. nucleatum inoculation increased DSS induced colitis. Neither $E$. rectale nor $F$. nucleatum inoculation can induce obvious colitis without DSS exposure. Group 'E. rectale+DSS' showed the highest histology score, suggesting that E. rectale increased DSS induced colitis. Group 'DSS' and 'F. nucleatum + DSS' showed similar histology score, -suggesting that F. nucleatum did not increase DSS-induced colitis.

Figure $4 \mathrm{~d}-\mathrm{g}$ showed that colon length from the distal end to the colon-cecum junction and spleen weight changes. E. rectale or $F$. nucleatum inoculation in water drinking mice did not induce a decrease in colon length or increase spleen weight. DSS exposure caused significant decrement in colon length or increment in spleen weight, and additional $E$. rectale inoculation further increased this effect. By contrast, F. nucleatum inoculation did not increase the effect of DSS on colon length shorten or spleen weight increment.

As shown in Fig. 4h and j, Body weight in inoculated groups showed no significant difference with Water group. Body weight in DSS group showed significant difference starting at day 10 , additional $F$. nucleatum inoculation shift this time point to day 9 , and additional E. rectale inoculation shift this time point to day 7 . Body weight in group ' $E$. rectale+DSS' continued to decrease until the end of experiment, but body weight in groups 'DSS' and group 'F. nucleatum + DSS' declined slowly from day 13 to the end of experiment, the reason of which may be due to $E$. rectale but not $F$. nucleatum inoculation enhance colitis induced by DSS. In addition, all mice survived until the end of experiment.

As shown in Fig. 4i and j, there are no occult or gross blood in stool in Water group and inoculated groups. DSS drinking groups had occult blood in stool at day 4 and gross blood in stool at day 5 . These groups began to exhibit gross blood in all stool at day 8. DSS group and 
F. nucleatum + DSS group both showed a decrease in gross blood and an increase in adult blood in stool from day 10. Occurrence of occult blood in stool of these two groups also continued to decrease from day 12 to the end of experiment. Compared to these two groups, $E$. rectale + DSS group has more time of gross blood occurrence, and incidence rate of occult blood decrease slower.

\section{E. rectale LPS promotes NF-KB p65 expression in nuclei of intestinal epithelial cells}

$\mathrm{NF}-\mathrm{kB}$ is a critical transcription regulator that regulates the expression of many genes of inflammatory functions and the immune system. NF- $\mathrm{kB}$ activation facilitates both tumor development and metastatic progression cancer $[15,16]$. NF-kB has two subunits, p50 and p65, to form a homodimer or heterodimer. The dimer maintains normal physiological function in vivo. In normal conditions, NF- $\mathrm{KB}$ is sequestered by members of the IкB family to form an inactivated compound in the cytoplasm. Under external stimuli, such as enteroinvasive bacterial infection or cytokine stimulation to intestinal epithelial cells $[17,18]$, NF- $\mathrm{kB}$ is activated. During this process, $\mathrm{I} \kappa \mathrm{B}$ kinase (IKK) is activated and caused $\mathrm{I}_{\kappa} \mathrm{B}$ degradation, which induced NF- $\mathrm{KB}$ dissociation from I $\mathrm{KB}$ and transferred to the nucleus. Therefore, we measured expression

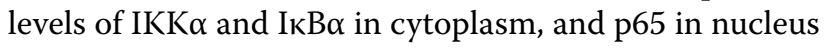
to determine NF-KB activation.

Two human normal colon epithelial cell lines, HCoEpiC and NCM460, were used to investigate whether E. rectale LPS induces inflammatory in vitro. Our results (Fig. 5) showed that NF- $\mathrm{KB}$ is activated in both $\mathrm{HCoEpiC}$ and NCM460 when they were treated by $20 \mathrm{ng} / \mathrm{ml}$ of $E$. rectale LPS for $2.5 \mathrm{~h}$. NF-kB was significantly expressed in nuclei of HCoEpiC and NCM460 cells after treating with $E$. rectale LPS. By contrast, $F$. nucleatum LPS does not show this effect at the same concentration. Purity measurement of LPS is shown in Additional file 1: Figure S4.

\section{Discussion}

According to the 'driver-passenger' model proposed by Tjalsma et al. [9], CRC 'driver' bacteria may not preferentially inhabit on-tumor sites, because many 'passenger' bacteria have high adaptability on-tumor sites and colonize most of the on-tumor sites niches. Conversely, the off-tumor site niches may be maintained similar to that on healthy intestinal tract. Thus, 'driver' bacteria may still be present in considerable abundance in off-tumor sites during CRC tumorigenesis [19]. We found that E. rectale distribution in our samples fit the characteristic of 'driver' bacteria, leading to our further investigation on the contribution of $E$. rectale to colitis in vitro and in vivo.

F. nucleatum is anaerobic Gram-negative bacterium, which is an adherent and invasive species. It is very suitable to survive in the broken colon wall and has high abundance in IBD [20] or CRC [6] samples. Because $F$. nucleatum is a potential 'passenger' bacterium [9], it is used as control bacterium in this study. Many reports found that $F$. nucleatum has higher abundance in CRC patients biopsy or fecal samples measured by $16 \mathrm{~S}$ ribosomal RNA profiling [21, 22]. Our results also exhibited that abundance of $F$. nucleatum increased in CRC patient samples, especially in on-tumor sites.

E. rectale is an anaerobic Gram-positive bacterium and is one of the most abundant bacterial species in human fecal samples. Some reports showed that E. rectale is a major species for butyrate formation, which is

\footnotetext{
(See figure on next page.)

Fig. 4 DSS induced colitis model of Balb/c mice. a Experimental design of seven-week-old Balb/c mouse model to evaluate the DSS exposure and bacterial inoculation. Six groups with different treatment were used, with each group having five mice. Body weight was measured daily, and organ was harvested immediately after euthanasia. $\mathbf{b}$ Representative images of H\&E stained colon sections showed effect of DSS and bacterial inoculation on histopathological changes. Colon tissue from groups 'Water,' 'E. rectale + water' and 'F. nucleatum + water' did not show any specific pathologic changes. Colon tissue from groups 'DSS' and 'F. nucleatum + DSS' showed rupture of the epithelium, segmental regions of marked acute inflammatory cell infiltration, ulceration, different size and shape of intestinal crypts, local destruction of crypts, which were indicated by arrows. Group 'E. rectale+DSS' had multiple crypts destruction and increased severity of the same histopathological changes seen in groups 'DSS' and 'F. nucleatum +DSS. c Graph indicated the histology scores of six groups. Data were presented as mean \pm STD. P-values were determined using the t-test. Other groups were compared with group 'Water'. Groups 'E. rectale + DSS' and 'F. nucleatum + DSS' were compare with group 'DSS'. * $P<0.05$ represents a significant difference from group 'Water', $\# P<0.05$ represents a significant difference from group 'DSS'. $\mathbf{d}-\mathbf{g}$ Effect of DSS and bacterial inoculation on colon length and spleen weight. Other groups were compared with group 'Water'. Groups 'E. rectale + DSS' and 'F. nucleatum + DSS' were compare with group 'DSS'. * $P<0.05$ represents a significant difference from group 'Water', \#P<0.05 represents a significant difference from group 'DSS'. d Representative gross images and measurements of mouse colons with cecums still attached. Arrows indicate the colon-cecum junction as a landmark for colon length measurement. e Diagram of colon length. Column indicated the mean \pm STD ( $n=5$ mice per group). $\mathbf{f}$ Gross picture of spleen. $\mathbf{g}$ Spleen weight measured at the time of harvested. Column indicated the mean \pm STD ( $\mathrm{n}=5 \mathrm{mice}$ per group). $\mathbf{h}-\mathbf{j}$ Body weight and stool grading of mice. Black arrow indicates the day of bacterial inoculation. Blue arrow indicates the day that mice began to drink water without DSS. Points show the mean from five mice or five stool samples, and standard deviations are shown as error bars. $\mathbf{h}$ Mice body weight alterations during the 17-day experiments. i Daily stool grading during the 17-day experiments. Stool grade 0 represents no occult or gross blood, stool grade 1 represents occult blood, stool grade 2 represents gross blood. j Other groups were compared with group 'Water' or group 'DSS'. Days represent significant difference from group 'Water' or group 'DSS' $(P<0.05)$. There are no significant difference among group 'Water,' $E$. rectale + water' and 'F. nucleatum + water'
} 


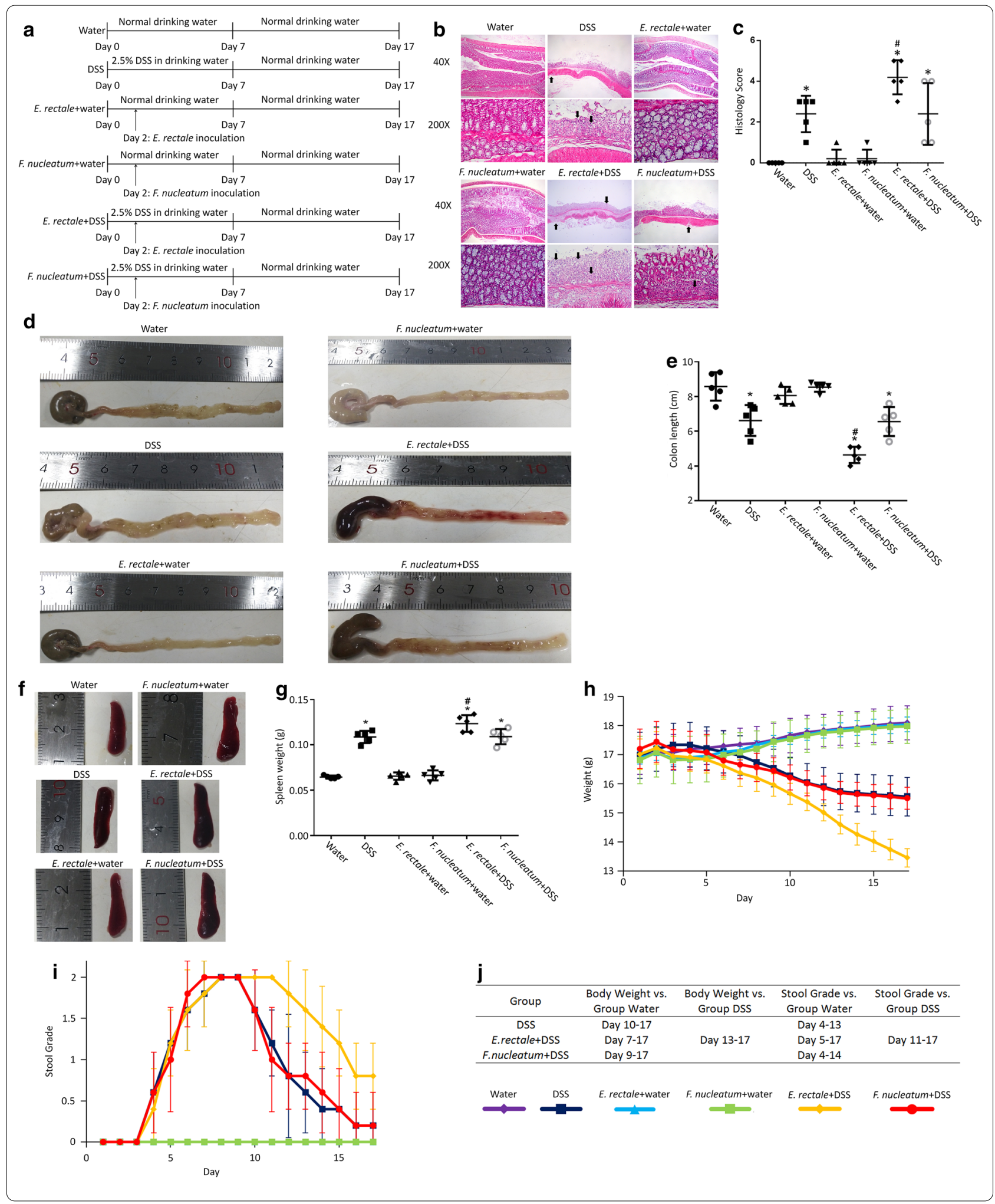




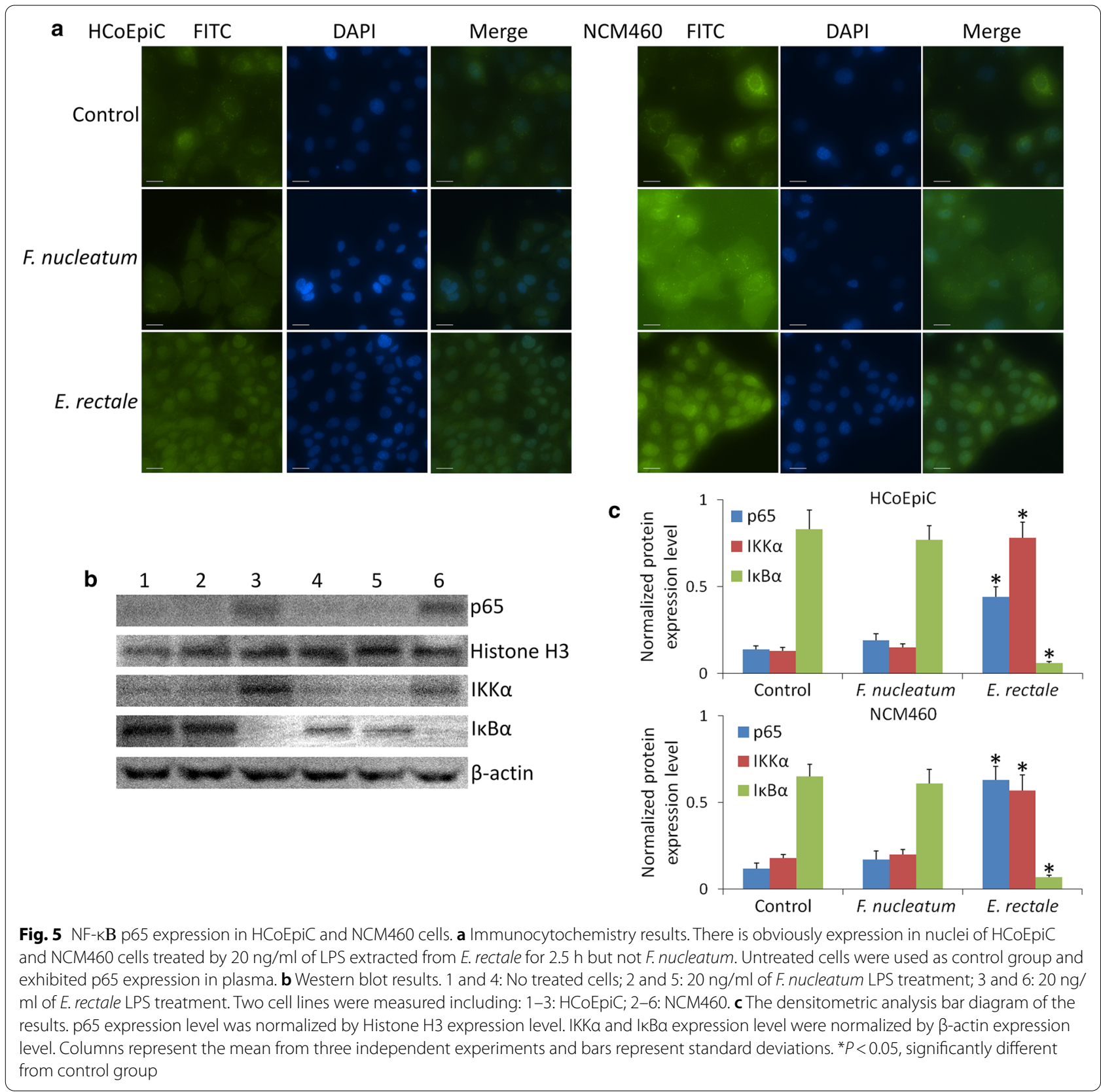

the preferred energy source for colonocytes and benefits for colon health [23], but butyrate also promotes CRC by stimulating the transformation of colonic epithelial cells and inducing aberrant proliferation [2]. E. rectale is reported to be significantly less present [24] in ulcerative colitis (UC) patients, which implies that E. rectale may initiate colitis that leads to CRC initiation if E. rectale is a 'driver' bacterium. According to this report, E. rectale seems to function as probiotic, but some recent studies that focused on distribution of intestinal microflora in CRC samples implied that $E$. rectale may be a potential 'driver' bacterium [7, 9]. Butyrate-producing bacteria, including Eubacterium, were found to be less abundant in CRC fecal microbiota measured by $16 \mathrm{~S}$ ribosomal RNA profiling [12]. To our best knowledge, the studies about E. rectale abundance in CRC samples measured by $16 \mathrm{~S}$ ribosomal RNA profiling are lacking. There are only a few reports [22] on which other species in the genus Eubacterium were measured by $16 \mathrm{~S}$ ribosomal RNA profiling in CRC samples, such as E. hallii, E. oxidoreducens and E. ruminantium, which exhibited different trend of abundance changes in carcinogenesis process, suggested that 
different species in the genus Eubacterium have different adaptabilities to the on-tumor site niche. Our $16 \mathrm{~S}$ ribosomal RNA profiling also showed that $E$. rectale has considerable density in healthy samples and higher abundance in off-tumor sites, but abundance of $E$. rectale declined in on-tumor sites significantly. Thus, we further investigated whether $E$. rectale contributes to CRC initiation by promoting inflammation in vitro and in vivo.

Cancer is a disease mainly caused by genetic mutations, but inflammation can temporarily bypass the mutation requirement for tumor initiation. Besides mutations, IBD is an important risk factor for the development of CRC. Two of the most common forms of IBD are Crohn's disease (CD) and ulcerative colitis (UC). For example, Crohn's disease (CD) increases cumulative risk of colitis associated cancer (CAC) by up to $8 \%$ [25], and Ulcerative colitis (UC) up to $18-20 \%$ risk of CAC [26]. Wnt/ $\beta$ catenin, which is a critical pathway to regulate normal and malignant cell proliferation, is activated by mutation in over $90 \%$ of sporadic CRC [27], which including adenomatous polyposis coli (APC). But several inflammatory pathways, including NF-kB, can induce Wnt/ $\beta$ catenin activation without any mutations in APC [28].

Some of the symbiotic or commensal intestinal bacteria in human beings and mice have been found as conditionally pathogenic, which induces various forms of IBD. For example, Helicobacter spp. infection in $\mathrm{Il}_{10}{ }^{-/-}$mice usually induces IBD and consequently CAC development [29]. Under normal conditions, intestinal bacteria are separated from immune system by complete protective epithelial barrier. When intestinal epithelial barrier is breakdown by some factors, for example DSS, commensal flora translocates to interact with immune system, which causes IBD development. Many potential 'driver' bacteria induce IBD, such as Bacteroides fragilis $[10,11]$ and Clostridium difficile [30]. Several reports show that abundance of Fusobacterium nucleatum [20] increases and $E$. rectale declines [14] in UC patients. Although $F$. nucleatum is also enriched in CRC samples and appears to be the most common passenger bacterium [5], this does not constitute sufficient evidence to confirm that it plays an active part in IBD or CRC progression [7]. And the relation between $E$. rectale and IBD promotion is also still unknown.

\section{Conclusion}

In our study, we investigated the relation between E. rectale and IBD promotion by utilizing the well-established dextran sulfate sodium (DSS) model of colitis in Balb/c mouse. This model was widely used to mimic characteristics of IBD, induce acute colitis and sustain chronic levels of inflammation [31]. This model has been further verified by applications of several therapeutic agents used to treat human IBD [32]. We found that E. rectale but not $F$. nucleatum prolonged and/or worsened the severity of DSS-induced colitis, and E. rectale also activated inflammatory factor, NF- $\mathrm{BB}$, in normal colon epithelial cells. Combined with $E$. rectale distribution in our samples, our findings suggest that $E$. rectale is a 'driver' bacterium and contribute to CRC initiation by promoting colitis.

\section{Supplementary Information}

The online version contains supplementary material available at https://doi. org/10.1186/s13099-020-00396-z.

Additional file 1. Additional figures.

\section{Abbreviations}

CRC: Colorectal cancer; IBD: Inflammatory bowel disease; PAMP: Pathogenassociated molecular patterns; LPS: Lipopolysaccharide; ETBF: B. fragilis; BFT: Metalloprotease toxin; LefSe: Linear discriminant analysis Effect Size; T:Tumor position; P: Para-tumor epithelia position; N: Normal epithelia position; H: Healthy people; DSS: Dextran sodium sulfate; CFU: Colony forming units; H\&E: Hematoxylin and eosin; LDA: Linear discriminant analysis; IKK: IKB kinase.

\section{Acknowledgements}

Not applicable.

\section{Authors' contributions}

YW performed the experiments of cells and animals. Xh. W performed the experiments of $16 s$ RNA sequencing. $\mathrm{Xj}$. W and CZ collected samples of gut mucosal microbiome. JL and SH wrote the manuscript. All authors read and approved the final manuscript.

\section{Funding}

This work was partially supported by the Natural Science Foundation of China Grant number 81972826, and the Fundamental Research Funds for the Central Universities, Nankai University Grant number 63191440, and Key R\&D Projects in the Tianjin Science and Technology Pillar Program Grant number 19YFZCSY00420, and National key R\&D Program of China Grant number 2017YFC1700604, and National key R\&D Program of China Grant number 2017YFC1700606, and Tianjin 131 Innovative Talent Training Project in 2018, and the Research Foundation of Tianjin Union Medical Center number 2018 YJ023.

\section{Availability of data and material}

The 165 rRNA sequencing reads have been submitted to the NCBI SRA database under accession number PRJNA606879. All the data are provided in this manuscript and supplementary materials.

\section{Ethics approval and consent to participate}

Research was conducted according to the principles expressed in the Declaration of Helsinki. The study was approved by the Ethics Committee of Tianjin Union Medical Center. Patients provided written informed consent for the collection of samples and subsequent analysis when required. All animal experiments complied with the National Institutes of Health guide for the care and use of laboratory animals (NIH Publications No. 8023, revised 1978).

\section{Consent for publication}

Not applicable.

\section{Competing interests}

All the authors have no conflict of interest.

\section{Author details}

${ }^{1}$ Laboratory of Oncologic Molecular Medicine, Tianjin Union Medical Center, Nankai University, No. 190 Jieyuan Rd., Hongqiao district, Tianjin 300121, China. ${ }^{2}$ TEDA Institute of Biological Sciences and Biotechnology, Nankai University, TEDA, Tianjin 300071, China. ${ }^{3}$ Advanced Studies in Genomics, Proteomics, 
and Bioinformatics, University of Hawaii At Manoa, 2538 McCarthy Mall, Snyder Hall, Honolulu, HI 96822, USA.

Received: 4 October 2020 Accepted: 21 December 2020

Published online: 12 January 2021

\section{References}

1. Fearon ER. Molecular genetics of colorectal cancer. Annu Rev Pathol. 2011:6:479-507.

2. Killeen SD, Wang JH, Andrews EJ, Redmond HP. Bacterial endotoxin enhances colorectal cancer cell adhesion and invasion through TLR-4 and NF-kB-dependent activation of the urokinase plasminogen activator system. Br J Cancer. 2009;100:1589-602.

3. Belcheva A, Irrazabal T, Robertson SJ, et al. Gut microbial metabolism drives transformation of Msh2-deficient colon epithelial cells. Cell. 2014;158:288-99.

4. Ijssennagger N, Belzer C, Hooiveld GJ, et al. Gut microbiota facilitates dietary heme-induced epithelial hyperproliferation by opening the mucus barrier in colon. Proc Natl Acad Sci USA. 2015;112:10038-43.

5. Kostic AD, Gevers D, Pedamallu CS, et al. Genomic analysis identifies association of Fusobacterium with colorectal carcinoma. Genome Res. 2011:22:292-8.

6. Tomkovich S, Yang Y, Winglee K, et al. Locoregional effects of microbiota in a preclinical model of colon carcinogenesis. Cancer Res. 2017;77:2620-32.

7. Castellarin M, Warren RL, Freeman JD, et al. Fusobacterium nucleatum infection is prevalent in human colorectal carcinoma. Genome Res. 2011;22:299-306.

8. Amitay EL, Werner $\mathrm{S}$, Vital M, et al. Fusobacterium and colorectal cancer: causal factor or passenger? Results from a large colorectal cancer screening study. Carcinogenesis. 2017;38:781-8.

9. Tjalsma H, Boleij A, Marchesi JR, et al. A bacterial driver-passenger model for colorectal cancer: beyond the usual suspects. Nat rev Microbiol. 2012;10:575-82.

10. Rhee KJ, Wu SG, Wu XQ, et al. Induction of persistent colitis by a human commensal, enterotoxigenic bacteroides fragilis, in wild-type C57BL/6 mice. Infect Immun. 2009;77:1708-18.

11. Rabizadeh S, Rhee K, Wu S, et al. Enterotoxigenic Bacteroides fragilis: a potential instigator of colitis. Inflamm Bowel Dis. 2007;13:1475-83.

12. Yang $T$, Lee $W$, Tu $S$, et al. Enterotype-based analysis of gut microbiota along the conventional adenoma-carcinoma colorectal cancer pathway. Sci Rep. 2019;9:10923.

13. Zhang $H$, Chang $Y$, Zheng $Q$, et al. Altered intestinal microbiota associated with colorectal cancer. Front Med. 2019:13:461-70.

14. Knoll RL, Forslund K, Kultima JR, et al. Gut microbiota differs between children with inflammatory bowel disease and healthy siblings in taxonomic and functional composition: a metage-nomic analysis. Am J Physiol Gastrointest Liver Physiol. 2017;312:G327-39.

15. Wullaert A, Bonnet MC, Pasparakis M. NF-kB in the regulation of epithelial homeostasis and inflammation. Cell Res. 2011;21:146-58.

16. Porta $C$, Larghi P, Rimoldi $M$, et al. Cellular and molecular pathways linking inflammation and cancer. Immunobiology. 2009;214:761-77.

17. Kim JM, Cho SJ, Oh YK, et al. Nuclear factor-kappa B activation pathway in intestinal epithelial cells is a major regulator of chemokine gene expression and neutrophil migration induced by Bacteroides fragilis enterotoxin. Clin Exp Immunol. 2002;130:59-66.

18. Elewaut $D$, DiDonato JA, Kim JM, et al. NF-kappa B is a central regulator of the intestinal epithelial cell innate immune response induced by infection with enteroinvasive bacteria. J Immunol. 1999;163:1457-66.

19. Marchesi JR, Dutilh BE, Hall N, et al. Towards the human colorectal cancer microbiome. PLoS ONE. 2011;6:e20447.
20. Yao P, Cui M, Wang H, et al. Quantitative analysis of intestinal flora of Uygur and Han ethnic Chinese patients with ulcer-ative colitis. Gastroenterol Res Pract. 2016;2016:9186232.

21. ShahID MS, DeSantis T, Yamal JM, et al. Re-purposing $16 \mathrm{~S}$ rRNA gene sequence data from within case paired tumor biopsy and tumor-adjacent biopsy or fecal samples to identify microbial markers for colorectal cancer. PLoS ONE. 2018;13:e0207002.

22. Mangifesta M, Mancabelli L, Milani C, et al. Mucosal microbiota of intestinal polyps reveals putative biomarkers of colorectal cancer. Sci Rep. 2018:8:13974.

23. Pryde $\mathrm{SE}$, Duncan $\mathrm{SH}$, Hold GL, et al. The microbiology of butyrate formation in the human colon. FEMS Microbiol Lett. 2002;217:133-9.

24. Li KY, Wang JL, Wei JP, et al. Fecal microbiota in pouchitis and ulcerative colitis. World J Gastroenterol. 2016:22:8929-39.

25. Canavan C, Abrams KR, Mayberry J. Meta-analysis: colorectal and small bowel cancer risk in patients with Crohn's disease. Aliment Pharmacol Ther. 2006;23:1097-104

26. Eaden JA, Abrams KR, Mayberry JF. The risk of colorectal cancer in ulcerative colitis: a metaanalysis. Gut. 2001;48:526-35.

27. Bienz M, Clevers H. Linking colorectal cancer to Wnt signaling. Cell. 2000;103:311-20

28. Kaler P, Godasi BN, Augenlicht L, et al. The NF-kappaB/AKT-dependent induction of Wnt signaling in colon cancer cells by macrophages and IL-1 beta. Cancer Microenviron. 2009;2:69.

29. Chichlowski M, Sharp JM, Vanderford DA, et al. Helicobacter typhlonius and Helicobacter rodentium differentially affect the severity of colon inflammation and inflammation-associated neoplasia in IL10-deficient mice. Comp Med. 2008;58:534-41.

30. Bien J, Palagani V, Bozko P. The intestinal microbiota dysbiosis and Clostridium difficile infection: is there a relationship with inflammatory bowel disease? Therap Adv Gastroenterol. 2013:6:53-68.

31. Melgar S, Karlsson A, Michaëlsson E. Acute colitis induced by dextran sulfate sodium progresses to chronicity in C57BL/6 but not in BALB/C mice: correlation between symptoms and inflammation. Am J Physiol Gastrointest Liver Physiol. 2005;288:G1328-38.

32. Melgar S, Karlsson L, Rehnström E, et al. Validation of murine dextran sulfate sodium-induced colitis using four therapeutic agents for human inflammatory bowel disease. Int Immunopharmacol. 2008:8:836-44.

33. Masella AP, Bartram AK, Truszkowski JM, et al. PANDAseq: paired-end assembler for illumina sequences. BMC Bioinformatics. 2012;13:31.

34. Caporaso JG, Kuczynski J, Stombaugh J, et al. QIIME allows analysis of high-throughput community sequencing data. Nat Methods. 2010;7:335-6. https://doi.org/10.1038/nmeth.f.303.

35. Segata N, Izard J, Waldron L, et al. Metagenomic biomarker discovery and explanation. Genome Biol. 2011;12:r60

36. Węglarz L, Parfiniewicz B, Mertas A, et al. Effect of endotoxins isolated from Desulfovibrio desulfuricans soil and intestinal strain on the secretion of TNF-a by human mononuclear cells. Polish J Environ Stud. 2006;15:615-22.

37. Shi G, Zheng X, Wu X, et al. All-trans retinoic acid reverses epithelial-mesenchymal transition in paclitaxel-resistant cells by inhibiting nuclear factor kappa B and upregulating gap junctions. Cancer Sci. 2019;110:379-88.

\section{Publisher's Note}

Springer Nature remains neutral with regard to jurisdictional claims in published maps and institutional affiliations. 